\title{
Research on the Development of Blockchain in Supply Chain
}

\author{
JunJie Zheng ${ }^{1 *}$, TianFeng Ye ${ }^{1}$ \\ ${ }^{1}$ Finance, Xiamen University International College
}

\begin{abstract}
At the present stage, China's economy is at a critical stage of transformation and development, and supply chain finance has also entered a deep stage of development. In order to promote supply chain finance to get rid of hollowing out and other traps, it is necessary to improve the way of financial information service. Some characteristics of blockchain technology are consistent with the development of supply chain finance, which can improve the industry's ability to control risks related to supply chain finance to a certain extent, and make supply chain finance better serve the real economy.On the basis of previous basic research, this paper creatively puts forward how to use blockchain to solve the problems existing in supply chain finance, and discusses in detail the obstacles to the popularization of blockchain technology in the field of supply chain finance, thus providing some schemes for realizing the application of blockchain in the field of supply chain finance.
\end{abstract}

\section{Introduction}

\section{1 research background}

Blockchain technology is the core component of electronic money. Relying on point-to-point network and distributed system timestamps, the network server can manage data independently and spontaneously. This technology also makes many electronic money successfully solve the problem of repeated consumption of money. With years of development, blockchain technology is developing continuously in many fields, and plays a great role in promoting supply chain finance.

The essence of supply chain finance is to help the core enterprises in the supply chain provide all-round credit services to the related enterprises at the upper and lower levels of their industries, thus reducing the operating costs of the whole supply chain. [1]Due to the integration and development of supply chain finance and real economy for many years, the structure of supply chain finance in China is relatively rigid, and there are some problems such as serious imbalance of credit distribution and financing difficulties for small and medium-sized enterprises.

Blockchain technology itself has many technical characteristics, which can provide pioneering methods in financing activities, reduce the cost of the whole small and medium-sized enterprises and greatly improve the capital turnover capacity of the whole manufacturing industry.

\section{2 research purpose}

Blockchain technology has many advantages, such as transaction security, transparency, low risk, etc. It has a lot of reference significance for the development of supply chain finance. However, in the field of supply chain finance, the application of blockchain technology mostly stays at the stage of theoretical and conceptual design, and there is a lack of systematic research on the follow-up development path.[2]

In view of the present situation of blockchain and the development of integration of supply chain finance and blockchain technology, this paper provides more solutions for supply chain finance to empower the real economy.

\section{3 research significance}

Theoretically, blockchain technology has five characteristics in essence, and all applications are developed around these five characteristics.[3] These characteristics also make blockchain technology more or less have certain application potential in any field in the world today.

From a realistic point of view, this paper fully analyzes the possibility of close connection between blockchain technology and supply chain finance, summarizes and puts forward the problems and solutions encountered in the popularization and practice of blockchain technology in the field of supply chain finance, which has important reference significance for promoting the rapid transformation of supply chain finance.

\section{Theoretical basis}

\section{1 theoretical basis of blockchain}

\subsection{1 decentralized structure}

*Corresponding author's e-mail: 32920182201283@stu.xmu.edu.cn 
The decentralized structure of blockchain is quite different from the centralized deployment mode of most databases. The interactive trust relationship between artificial scenes and data is constructed by mainly adopting multi-node, decentralized nodes and distributed deployment mode, and using the relevant theoretical methods of cryptography. When a node completes the calculation of recording rate, other nodes will synchronize information accordingly to ensure that different nodes have consistent data.[4] This structure of blockchain can ensure the safety of data and information, and at the same time, it can minimize the probability of data tampering.

\subsection{2 data information can not be tampered with}

By forming a one-way chain structure, blockchain contains multiple information of several transactions in each block, and the next block includes the ID and digital signature of the previous block. Once the data in a block is tampered, its digital signature will be renamed, which ensures that the historical transaction data of blockchain will not be tampered.

\subsection{3 distributed accounting and storage}

The accounting process of blockchain is a process of single node accounting and synchronization of other nodes, and the whole network stores and backs up the same information in a distributed way. The advantage of this accounting process is that even if some nodes in the network are abnormal, it will not affect the accounting and storage of other nodes.

\subsection{4 smart contracts can be programmed flexibly}

Smart contracts save the performance commitments involved in business in the form of codes and computer programs, and different business forms can sign different smart contracts. This technology is the foundation of building a distrustful trading environment in blockchain.

\section{2 theoretical basis of supply chain finance}

\subsection{1 the concept of supply chain finance}

With the intensification of market competition and international trade, the competition among individual enterprises has changed from local competition in supply chain to game between industrial chains, and the interdependence among multiple parties in the unified supply chain is also increasing. Generally speaking, supply chain finance is a liquidity property management method based on core customers, real trade as a precondition, cash flow self-compensation decomposition method based on closed system, pledge registration according to the technical nature of accounts receivable, management and control by a third party, and aiming at providing comprehensive financial services to upstream and downstream enterprises in the supply chain.

\subsection{2 characteristics of supply chain finance}

(1) Generally speaking, intelligent supply chain management is the basic core concept of supply chain financial services, and the quality and reliability of intelligent chain operation will directly affect the scale and risk of supply chain finance.

(2) Internet big data. A comprehensive review of customer data with the help of Internet big data is the premise of supply chain financial services, and whether it meets the service standards is judged through the review.

(3) Closed system. Closed system is a mandatory requirement for the operation of supply chain financial services. Through the closed system management, the overall goal of risk control in the whole process is achieved.

(4) Lack of liquidity. Generally speaking, the lack of liquidity of various forms of asset settlement units caused by production and trade is an important reason for the slow development of supply chain finance. How to revitalize these assets has become an urgent need for the development of supply chain finance.

\section{Application status and suggestions of blockchain}

\subsection{The status quo of blockchain application in supply chain finance}

With the comprehensive development of blockchain in many fields, the financial value chain of supply chain has been effectively reshaped by means of blockchain, and its innovative applications have gradually increased since its development. [5] These applications also make blockchain go far beyond the scope of technological change, and make the original business model fundamentally changed.

From the perspective of service mode, there are about four main transaction modes in supply chain finance.

(1) Bank-led mode. This model is gradually weakening, but from the overall layout, many Chinese banks have launched supply chain financial products based on blockchain. However, there are fewer and fewer bank-led models at present, because banks themselves are not the demand side of funds, but are supported by traditional businesses, and their motivation is insufficient.

(2) Internet finance company+blockchain company cooperation mode

By providing technology through blockchain, Internet finance companies rely on known customers in real industries to transfer the credit of core enterprises to other small and medium-sized enterprises through blockchain technology.

(3) Self-built mode of entity enterprises

Some entities rely on their own strength to build a free supply chain financial service platform based on blockchain technology.

(4) Cooperative development mode between entity enterprises and blockchain companies

To a certain extent, Internet finance companies have little value in the field of supply chain finance. Blockchain companies can directly connect with industrial giants to 
realize joint development. At present, this model is also rising.

\subsection{Blockchain in the field of supply chain finance}

\subsubsection{Lack of supporting legal supervision and supervision subjects}

At present, the existing laws and regulations are not perfect, mainly because the application of blockchain in supply chain is growing too fast, which leads to the failure to promote the smooth development of blockchain technology in supply chain finance.

\subsubsection{There are bottlenecks in technology}

Although the integration of blockchain technology and supply chain finance is a great breakthrough at the technical level, the data and information nodes of blockchain technology itself may be cracked by more advanced algorithms, and the hash function solves this problem to a certain extent, unless the number of changed nodes exceeds $51 \%$. However, in terms of probability, it is possible to decipher $51 \%$.[6] When the input cost is large enough, $51 \%$ of nodes may be compromised. In other words, blockchain technology still has certain risks in ensuring the security of data and information.

However, when an important smart contract technology in blockchain technology is applied to Ethereum, the problem that the loopholes caused by smart contract cannot be repaired in time, which leads to the problem that a large number of Ethereum are stolen. Therefore, only when the technology is quite perfect can the occurrence of hidden dangers be minimized.

\section{Conclusions and prospects}

\subsection{Conclusions}

The mode of blockchain+supply chain finance should develop in the direction of four flows in one, visual management of the whole process, digitization of assets and bills, omni-directional dynamic supervision, and establishment of a comprehensive risk prevention and control network. This kind of norm is an important guide and standard for the development of blockchain financial services. Under this development direction, the accessibility, credibility and convenience of supply chain financial services can be greatly improved.

Core enterprises play the most important role in this model. The core of supply chain financial services lies in digitalizing accounts payable of core enterprises and forming negotiable electronic payment vouchers. Only when the core final acceptance credit is formally driven can the penetrating transmission of credit value to the whole supply chain be formed. Therefore, core enterprises should continuously improve the healthy development of blockchain+supply chain finance by developing technology and promoting the integration of blockchain and cross-border settlement.

\subsection{Outlook}

Compared with supply chain finance, the development cycle of blockchain technology is relatively short, but its unique technical characteristics and technical guidelines have solved many problems in the financial field to a great extent, and supply chain finance has also become a major issue in the financial field.

After experiencing the prosperity and collapse of virtual currency, blockchain technology will gradually develop steadily under the supervision of laws and regulatory agencies. I believe that in the future, blockchain+supply chain financial technology will truly benefit most enterprises and realize financial empowerment of the real economy.

\section{References}

1. Song Hua, Huang Qian-yuan, Yang Yudong. The influence of financial orientation and supply chain finance on enterprise performance [J]. Journal of Management, 2021,18(05):760-768.

2. Song Hua, Huang Qianyuan, Yang Yudong. The influence of financial orientation and supply chain finance on enterprise performance $[\mathrm{J}]$. Journal of Management, 2021,18(05):760-768.

3. Yang Nong. Thoughts on the development of "blockchain+supply chain finance" [N]. Financial Times, 2021-04-19(010).

4. $\mathrm{Wu}$ huilan. analysis of the development trend of supply chain finance with the help of new technology $[\mathrm{J}]$. journal of science and technology economy, 2021,29(11):225-226.

5. Yu Haijing. Research on the Development Path of Supply Chain Finance Innovation of Commercial Banks under internet plus [D]. Wuhan University of Technology, 2018.

6. $\mathrm{Fu}$ Yonggui. Research on information sharing mechanism and management mode of supply chain based on blockchain [D]. Central University of Finance and Economics, 2018. 